\title{
Subdifferential inclusions and quasi-static hemivariational inequalities for frictional viscoelastic contact problems
}

\section{Stanisław Migórski ${ }^{1 *}$}

1 Institute of Computer Science, Faculty of Mathematics and Computer Science, Jagiellonian University, Łojasiewicza 6 , 30-348 Kraków, Poland

Received 1 March 2012; accepted 27 June 2012

\begin{abstract}
We survey recent results on the mathematical modeling of nonconvex and nonsmooth contact problems arising in mechanics and engineering. The approach to such problems is based on the notions of an operator subdifferential inclusion and a hemivariational inequality, and focuses on three aspects. First we report on results on the existence and uniqueness of solutions to subdifferential inclusions. Then we discuss two classes of quasi-static hemivariational ineqaulities, and finally, we present ideas leading to inequality problems with multivalued and nonmonotone boundary conditions encountered in mechanics.

MSC: $\quad 34 G 20,35 A 15,35 J 85,35 K 90,35 K 85,35 L 85,35 K 55,49 J 40,49 K 40,74 A x x$

Keywords: Hemivariational inequality $\cdot$ Subdifferential $\cdot$ Quasi-static $\cdot$ Multifunction $・$ Nonconvex $・$ Viscoelasticity

(C) Versita Sp. z o.o.
\end{abstract}

\section{Introduction}

The paper is a shortened version of the original parts of an invited talk presented by the author at the Sixth Symposium on Nonlinear Analysis (SNA2011) held in Toruń, Poland, September 7-9, 2011. The goal is to review and report on the results of recent studies on problems involving operator subdifferential inclusions in Banach spaces, quasi-static hemivariational inequalities, and their applications.

The notion of hemivariational inequality was introduced by P.D. Panagiotopoulos in the early 1980s, cf. [17, 18], as a generalization of the variational inequality. The hemivariational inequality formulation exploits the notion of the generalized gradient of Clarke-Rockafellar, cf. [1]. The latter was introduced for a class of locally Lipschitz functions and

*E-mail: stanislaw.migorski@ii.uj.edu.pl 
allows to give variational formulations of several mechanical phenomena involving the nonconvex and nondifferentiable energy functionals. Thus the theory of hemivariational inequalities provides mathematical results which arise and are applicable to variational theory of engineering problems involving nonmonotone multivalued relations. In the stationary case the hemivariational inequalities can be formulated as the substationary point problems for the corresponding nondifferentiable, nonsmooth and nonconvex energy functionals. The formulations of mechanical problems as hemivariational inequalities allow to give positive answers to unsolved or partially unsolved problems, cf. $[9-12,15,16]$. More information on modeling and analysis of contact phenomena can be found in $[5,7,8,20]$.

The goal of this paper is to give a survey on modeling of contact problems with nonsmooth potentials and on analysis of quasi-static hemivariational inequalities. We have tried to keep the presentation as simple as possible and this makes it accessible also to the newcomer in this field.

The content of the paper is as follows. We consider two classes of subdifferential inclusions in a framework of evolution triple of spaces. The first class involves inclusions with a history-dependent term for which we provide an existence and uniqueness result. The proof is based on arguments on pseudomonotone operators and fixed point. Then we specialize this result in the study of a class of history-dependent hemivariational inequalities. Such kind of problems arises in a large number of mathematical models which describe quasistatic processes of contact between a deformable body and an obstacle. To provide an example we consider a viscoelastic problem in which the frictional contact is modeled with subdifferential boundary conditions. We prove that this contact problem leads to a history-dependent hemivariational inequality in which the unknown is the velocity field. Then we apply our result in order to prove the unique weak solvability of the corresponding contact problem. In the second class of inclusions, we consider time-dependent possibly nonconvex nonsmooth functions and their Clarke subdifferentials operating on the unknown function. First we prove the existence of a weak solution. Then we study the asymptotic behavior of a sequence of solutions when a small parameter in the inertial term tends to zero. We prove that the limit function is a solution of a parabolic hemivariational inequality. Finally, we give applications to quasi-static viscoelastic frictional contact problems.

\section{Preliminaries}

In this section we recall some definitions we shall use in this paper, see e.g. Zeidler [21] and Denkowski et al. [2, 3]. Given a Banach space $X$, we denote its norm by $\|\cdot\|_{X}$. The dual space is denoted by $X^{*}$ and $\langle\cdot, \cdot\rangle_{X^{*} \times X}$, denoted also by $\langle\cdot, \cdot\rangle$, is the duality pairing between $X$ and $X^{*}$. The symbol $w-X$ is used for the space $X$ endowed with its weak topology. The notation $\mathcal{L}(X, Y)$ stands for the space of linear bounded operators from a Banach space $X$ to a Banach space $Y$. Also, we denote by $2^{X}$ the collection of all subsets of $X$. For $U \subset X$, we also write $\|U\|_{X}=\sup \left\{\|u\|_{X}: u \in U\right\}$. Let $\theta: X \rightarrow \mathbb{R}$ be a locally Lipschitz function. The generalized directional derivative of $\theta$ at $x \in X$ in the direction $v \in X$ is defined by

$$
\theta^{0}(x ; v)=\limsup _{y \rightarrow x, \lambda \downarrow 0} \frac{\theta(y+\lambda v)-\theta(y)}{\lambda} .
$$

The generalized gradient of $\theta$ at $x$ is the subset of $X^{*}$ given by

$$
\partial \theta(x)=\left\{\zeta \in X^{*}: \theta^{0}(x ; v) \geq\langle\zeta, v\rangle, v \in X\right\} .
$$

A locally Lipschitz function $\theta$ is called regular (in the sense of Clarke, cf. [1]) at $x \in X$ if for all $v \in X$ the one-sided directional derivative $\theta^{\prime}(x ; v)$ exists and satisfies $\theta^{0}(x ; v)=\theta^{\prime}(x ; v)$ for all $v \in X$.

Let $T: X \rightarrow 2^{X^{*}}$. An operator $T$ is said to be pseudomonotone if it satisfies the following conditions:

(a) for every $x \in X, T X$ is a nonempty, convex and weakly compact set in $X^{*}$;

(b) $T$ is u.s.c. on every finite dimensional subspace of $X$ into $X^{*}$ endowed with the weak topology; and

(c) if $x_{n} \rightarrow x$ weakly in $X, x_{n}^{*} \in T x_{n}$ and $\lim \sup \left\langle x_{n}^{*}, x_{n}-x\right\rangle \leq 0$, then for each $z \in X$ there exists $x^{*}(z) \in T x$ such that $\left\langle x^{*}(z), x-z\right\rangle \leq \liminf \left\langle x_{n}^{*}, x_{n}-z\right\rangle$. 
Let $L: D(L) \subset X \rightarrow X^{*}$ be a linear densely defined maximal monotone operator. An operator $T$ is said to be $p s e u-$ domonotone with respect to $D(L)$ (shortly L-pseudomonotone) if and only if (a) and (b) hold and

(d) if $\left\{x_{n}\right\} \subset D(L)$ is such that $x_{n} \rightarrow x$ weakly in $X, x \in D(L), L x_{n} \rightarrow L x$ weakly in $X^{*}, x_{n}^{*} \in T x_{n}, x_{n}^{*} \rightarrow x^{*}$ weakly in $X^{*}$ and $\lim \sup \left\langle x_{n}^{*}, x_{n}\right\rangle \leq\left\langle x^{*}, x\right\rangle$, then $x^{*} \in T x$ and $\left\langle x_{n}^{*}, x_{n}\right\rangle \rightarrow\left\langle x^{*}, x\right\rangle$.

An operator $T$ is said to be coercive if there exists a function $c: \mathbb{R}^{+} \rightarrow \mathbb{R}$ with $c(r) \rightarrow \infty$ as $r \rightarrow \infty$ such that $\left\langle x^{*}, x\right\rangle \geq c\left(\|x\|_{X}\right)\|x\|_{x}$ for every $\left(x, x^{*}\right) \in \operatorname{Graph}(T)$.

A single-valued operator $T: X \rightarrow X^{*}$ is said to be hemicontinuous, if for all $u, v, w \in X$ the functional $t \mapsto\langle T(u+t v), w\rangle$ is continuous on $[0,1] . T: X \rightarrow X^{*}$ is pseudomonotone if for each sequence $\left\{x_{n}\right\} \subseteq X$ such that it converges weakly to $x_{0} \in X$ and $\lim \sup \left\langle T x_{n}, x_{n}-x_{0}\right\rangle \leq 0$, we have $\left\langle T x_{0}, x_{0}-x\right\rangle \leq \liminf \left\langle T x_{n}, x_{n}-x\right\rangle$ for all $x \in X$.

Finally, we recall the following surjectivity result, cf. [2, Theorem 1.3.73], for multivalued operators which are pseudomonotone with respect to $D(L)$.

\section{Proposition 2.1.}

If $X$ is a reflexive, strictly convex Banach space, $L: D(L) \subset X \rightarrow X^{*}$ is a linear densely defined maximal monotone operator and $T: X \rightarrow 2^{X^{*}} \backslash\{\emptyset\}$ is bounded, coercive and pseudomonotone with respect to $D(L)$, then the operator $L+T$ is surjective.

\section{History-dependent subdifferential inclusions}

Let $\Omega \subset \mathbb{R}^{d}$ be an open bounded subset of $\mathbb{R}^{d}$ with a Lipschitz continuous boundary $\partial \Omega$ and $\Gamma \subseteq \partial \Omega, d=1,2,3$. Let $V$ be a closed subspace of $H^{1}\left(\Omega ; \mathbb{R}^{s}\right), s \geq 1, H=L^{2}\left(\Omega ; \mathbb{R}^{s}\right)$ and $Z=H^{\delta}\left(\Omega ; \mathbb{R}^{s}\right)$ with a fixed $\delta \in(1 / 2,1)$. Denoting by $i: V \rightarrow Z$ the embedding, by $\gamma: Z \rightarrow L^{2}\left(\Gamma ; \mathbb{R}^{s}\right)$ and $\gamma_{0}: H^{1}\left(\Omega ; \mathbb{R}^{s}\right) \rightarrow H^{1 / 2}\left(\Gamma ; \mathbb{R}^{s}\right) \subset L^{2}\left(\Gamma ; \mathbb{R}^{s}\right)$ the trace operators, we get $\gamma_{0} v=\gamma(i v)$ for all $v \in V$. For simplicity, in what follows, we omit the notation of the embedding $i$ and we write $\gamma_{0} v=\gamma v$ for all $v \in V$. It is well known from the theory of Sobolev spaces, cf. [2, 3, 21], that $\left(V, H, V^{*}\right)$ and $\left(Z, H, Z^{*}\right)$ form evolution triples of spaces and the embedding $V \subset Z$ is compact. We denote by $c_{e}$ the embedding constant of $V$ into $Z$, by $\|\gamma\|$ the norm of the trace in $\mathcal{L}\left(Z, L^{2}\left(\Gamma ; \mathbb{R}^{s}\right)\right)$ and by $\gamma^{*}: L^{2}\left(\Gamma ; \mathbb{R}^{s}\right) \rightarrow Z^{*}$ the adjoint operator to $\gamma$. We also introduce the following spaces:

$$
V=L^{2}(0, T ; V), \quad Z=L^{2}(0, T ; Z) \quad \text { and } \quad \widehat{\mathcal{H}}=L^{2}(0, T ; H)
$$

where $0<T<+\infty$. Since the embeddings $V \subseteq Z \subseteq H \subseteq Z^{*} \subseteq V^{*}$ are continuous, it is known that the embeddings $\mathcal{V} \subseteq Z \subseteq \widehat{\mathcal{H}} \subseteq Z^{*} \subseteq \mathcal{V}^{*}$ are also continuous, where $Z^{*}=L^{2}\left(0, T ; Z^{*}\right)$ and $\mathcal{V}^{*}=L^{2}\left(0, T ; V^{*}\right)$

Let $A:(0, T) \times V \rightarrow V^{*}, \mathcal{S}: \mathcal{V} \rightarrow \mathcal{V}^{*}, J:(0, T) \times L^{2}\left(\Gamma ; \mathbb{R}^{s}\right) \rightarrow \mathbb{R}$ and $f:(0, T) \rightarrow V^{*}$ be given. We consider the following inclusion of subdifferential form.

Problem 3.1.

Find $u \in V$ such that

$$
A(t, u(t))+\mathcal{S} u(t)+\gamma^{*} \partial J(t, \gamma u(t)) \ni f(t) \quad \text { for a.e. } \quad t \in(0, T)
$$

We note that in (1) the notation $\mathcal{S} u(t)$ stands for $(\mathcal{S} u)(t)$, i.e. $\mathcal{S} u(t)=(\mathcal{S} u)(t)$ for all $u \in \mathcal{V}$ and a.e. $t \in(0, T)$. The symbol $\partial J$ denotes the Clarke subdifferential of a locally Lipschitz function $J(t, \cdot)$. We adopt the following definition.

\section{Definition 3.2.}

A function $u \in \mathcal{V}$ is called a solution to Problem 3.1 if and only if there exists $\zeta \in Z^{*}$ such that for a.e. $t \in(0, T)$,

$$
A(t, u(t))+\mathcal{S} u(t)+\zeta(t)=f(t) \quad \text { and } \quad \zeta(t) \in \gamma^{*} \partial J(t, \gamma u(t))
$$


In order to state a result on the solvability of Problem 3.1, we need the following hypotheses.

I. $A:(0, T) \times V \rightarrow V^{*}$ is such that

(I.a) $A(\cdot, v)$ is measurable on $(0, T)$ for all $v \in V$;

(I.b) $A(t, \cdot)$ is hemicontinuous and strongly monotone for a.e. $t \in(0, T)$, i.e., $\left\langle A\left(t, v_{1}\right)-A\left(t, v_{2}\right), v_{1}-v_{2}\right\rangle_{v^{*} \times V} \geq$ $m_{1}\left\|v_{1}-v_{2}\right\|_{V}^{2}$ for all $v_{1}, v_{2} \in V$ with $m_{1}>0$;

(l.c) $\|A(t, v)\|_{v^{*}} \leq a_{0}(t)+a_{1}\|v\|_{v}$ for all $v \in V$, a.e. $t \in(0, T)$ with $a_{0} \in L^{2}(0, T), a_{0} \geq 0$ and $a_{1}>0$;

(l.d) $A(t, 0)=0$ for a.e. $t \in(0, T)$.

II. $\mathcal{S}: \mathcal{V} \rightarrow \mathcal{V}^{*}$ is such that

$$
\left\|\mathcal{S} u_{1}(t)-\mathcal{S} u_{2}(t)\right\|_{V^{*}} \leq L_{\mathcal{S}} \int_{0}^{t}\left\|u_{1}(s)-u_{2}(s)\right\|_{v} d s
$$

for all $u_{1}, u_{2} \in \mathcal{V}$, a.e. $t \in(0, T)$ with $L_{s}>0$.

III. $J:(0, T) \times L^{2}\left(\Gamma ; \mathbb{R}^{s}\right) \rightarrow \mathbb{R}$ is such that

(III.a) $J(\cdot, u)$ is measurable on $(0, T)$ for all $u \in L^{2}\left(\Gamma ; \mathbb{R}^{s}\right)$

(III.b) $J(t, \cdot)$ is locally Lipschitz on $L^{2}\left(\Gamma ; \mathbb{R}^{s}\right)$ for a.e. $t \in(0, T)$;

(III.c) $\|\partial J(t, u)\|_{L^{2}\left(\Gamma ; \mathbb{R}^{s}\right)} \leq c_{0}+c_{1}\|u\|_{L^{2}\left(\Gamma ; \mathbb{R}^{s}\right)}$ for all $u \in L^{2}\left(\Gamma ; \mathbb{R}^{s}\right)$, a.e. $t \in(0, T)$ with $c_{0}, c_{1} \geq 0$;

(III.d) $\left(z_{1}-z_{2}, u_{1}-u_{2}\right)_{L^{2}\left(\Gamma ; \mathbb{R}^{s}\right)} \geq-m_{2}\left\|u_{1}-u_{2}\right\|_{L^{2}\left(\Gamma ; \mathbb{R}^{s}\right)}^{2}$ for all $z_{i} \in \partial J\left(t, u_{i}\right), u_{i}, z_{i} \in L^{2}\left(\Gamma ; \mathbb{R}^{s}\right), i=1,2$, a.e. $t \in(0, T)$, $m_{2} \geq 0$;

(III.e) $J^{0}(t, u ;-u) \leq d_{0}\left(1+\|u\|_{L^{2}\left(\Gamma ; \mathbb{R}^{s}\right)}\right)$ for all $u \in L^{2}\left(\Gamma ; \mathbb{R}^{s}\right)$, a.e. $t \in(0, T)$ with $d_{0} \geq 0$.

We remark that condition II is satisfied for the operator $\mathcal{S}: \mathcal{V} \rightarrow \mathcal{V}^{*}$ given by

$$
\mathcal{S} v(t)=R\left(t, \int_{0}^{t} v(s) d s+v_{0}\right) \quad \text { for all } \quad v \in \mathcal{V}, \quad \text { a.e. } \quad t \in(0, T)
$$

where $R:(0, T) \times V \rightarrow V^{*}$ is such that $R(\cdot, v)$ is measurable on $(0, T)$ for all $v \in V, R(t, \cdot)$ is a Lipschitz continuous operator for a.e. $t \in(0, T)$ and $v_{0} \in V$. It is also satisfied for the Volterra operator $\mathcal{S}: \mathcal{V} \rightarrow \mathcal{V}^{*}$ given by

$$
\mathcal{S} v(t)=\int_{0}^{t} C(t-s) v(s) d s \quad \text { for all } \quad v \in \mathcal{V}, \quad \text { a.e. } \quad t \in(0, T),
$$

where $C \in L^{\infty}\left(0, T ; \mathcal{L}\left(V, V^{*}\right)\right)$. Clearly, in the case of the operators (2) and (3) the current value $\mathcal{S} v(t)$ at the moment $t$ depends on the history of the values of $v$ at the moments $0 \leq s \leq t$ and, therefore, we refer to the operators of the form (2) or (3) as history-dependent. We extend this definition to all the operators $\mathcal{S}: \mathcal{V} \rightarrow \mathcal{V}^{*}$ which satisfy condition II and, for this reason, we say that the subdifferential inclusions of the form (1) are history-dependent subdifferential inclusions. The main feature of such inclusions consists in the fact that they contain operators which, at any moment $t \in(0, T)$ depend on the history of the solution up to the moment $t$. This feature makes the difference with respect to the time-dependent subdifferential inclusions studied in literature in which, usually, the operators involved are assumed to depend on the current value of the solution $u(t)$.

In order to prove the existence and uniqueness for Problem 3.1, we first state a result on the unique solvability of a subdifferential inclusion in which the time variable plays the role of a parameter.

\section{Lemma 3.3.}

Assume that I holds and $f \in \mathcal{V}^{*}$. If one of the following hypotheses:

(i) (III.a)-(III.d) and $m_{1}>\max \left\{c_{1}, m_{2}\right\} c_{e}^{2}\|\gamma\|^{2}$,

(ii) III and $m_{1}>m_{2} c_{e}^{2}\|\gamma\|^{2}$ 
is satisfied, then the problem

$$
A(t, u(t))+\gamma^{*} \partial J(t, \gamma u(t)) \ni f(t) \quad \text { for a.e. } \quad t \in(0, T)
$$

has a unique solution $u \in \mathcal{V}$.

Proof. We provide main steps of the proof without details. First, since the operator $A(t, \cdot)$ is strongly monotone and $A(t, 0)=0$ for a.e. $t \in(0, T)$, it follows that $A(t, \cdot)$ is coercive with $m_{1}>0$, i.e. $\langle A(t, v), v\rangle_{V^{*} \times V} \geq m_{1}\|v\|_{V}^{2}$ for all $v \in V$, a.e. $t \in(0, T)$. Moreover, since the operator $A(t, \cdot)$ satisfies (I.b)-(I.c), it is pseudomonotone for a.e. $t \in(0, T)$. This follows from the facts that every strongly monotone operator is monotone and every bounded, hemicontinuous and monotone operator is pseudomonotone, cf. [21, Proposition 27.6]. Next, we define the operator $B:(0, T) \times V \rightarrow 2^{Z^{*}}$ by $B(t, v)=\gamma^{*} \partial J(t, \gamma v)$ for all $v \in V$, a.e. $t \in(0, T)$. Under either the hypothesis (i) or (ii), we can establish the following properties of the operator $B$.

(IV.a) $B(\cdot, v)$ is measurable for all $v \in V$;

(IV.b) $\|B(t, v)\|_{Z^{*}} \leq b_{0}\left(1+\|v\|_{v}\right)$ for all $v \in V$, a.e. $t \in(0, T)$ with $b_{0}>0$;

(IV.c) for all $v \in V$ and a.e. $t \in(0, T), B(t, v)$ is nonempty, convex, weakly compact subset of $Z^{*}$;

(IV.d) $\langle B(t, v), v\rangle_{V^{*} \times v} \geq-b_{1}\|v\|_{v}^{2}-b_{2}\|v\|_{v}-b_{3}$ for all $v \in V$, a.e. $t \in(0, T)$ with $b_{1}, b_{2}, b_{3} \geq 0$;

(IV.e) the graph of $B(t, \cdot)$ is closed in $Z \times\left(w-Z^{*}\right)$ topology for a.e. $t \in(0, T)$, i.e. if $\zeta_{n}(t) \in B\left(t, v_{n}\right)$ with $v_{n}, v \in V$, $v_{n} \rightarrow v$ in $Z$ and $\zeta_{n}(t), \zeta(t) \in Z^{*}, \zeta_{n}(t) \rightarrow \zeta(t)$ weakly in $Z^{*}$, then $\zeta(t) \in B(t, v)$.

Subsequently, we define the multivalued map $\mathcal{F}:(0, T) \times V \rightarrow 2^{V^{*}}$ by $\mathcal{F}(t, v)=A(t, v)+B(t, v)$ for all $v \in V$ and a.e. $t \in(0, T)$. From (l.a) and (IV.a), it is clear that $\mathcal{F}(\cdot, v)$ is a measurable multifunction for all $v \in V$. Exploiting [3, Proposition 6.3.66], we show that $\mathcal{F}(t, \cdot)$ is pseudomonotone and coercive for a.e. $t \in(0, T)$. Therefore, applying the fundamental surjectivity result, cf. e.g. [3, Theorem 6.3.70], it follows that $\mathcal{F}(t, \cdot)$ is surjective. This implies that for a.e. $t \in(0, T)$ there exists a solution $u(t) \in V$ of the problem (4). Furthermore, owing to the coercivity of $\mathcal{F}(t, \cdot)$, we deduce the following estimate:

$$
\|u(t)\|_{v} \leq c\left(1+\|f(t)\|_{v^{*}}\right) \quad \text { for a.e. } t \in(0, T) \text { with } \quad c>0 .
$$

Using the strong monotonicity of $A(t, \cdot)$, (III.d) and the hypothesis $m_{1}>m_{2} c_{e}^{2}\|\gamma\|^{2}$, we prove now that the solution to the problem (4) is unique. Also, we are able to prove that the solution of the problem (4) is a measurable function on $(0, T)$. Since $f \in \mathcal{V}^{*}$, from the estimate (5), we conclude that $u \in \mathcal{V}$ and (4) hold, which completes the proof of the lemma.

The existence and uniqueness result for Problem 3.1 reads as follows.

Theorem 3.4.

Assume I, II and $f \in \mathcal{V}^{*}$. If either (i) or (ii) of the hypothesis of Lemma 3.3 holds, then Problem 3.1 has a unique solution.

Proof. We use a fixed point argument. Let $\eta \in \mathcal{V}^{*}$. We denote by $u_{\eta} \in \mathcal{V}$ the solution of the following problem:

$$
A\left(t, u_{\eta}(t)\right)+\gamma^{*} \partial J\left(t, \gamma u_{\eta}(t)\right) \ni f(t)-\eta(t) \quad \text { for a.e. } \quad t \in(0, T)
$$

By Lemma 3.3 we know that $u_{\eta} \in \mathcal{V}$ exists and is unique. Next, we consider the operator $\Lambda: \mathcal{V}^{*} \rightarrow \mathcal{V}^{*}$ defined by

$$
\wedge \eta(t)=S u_{\eta}(t) \quad \text { for all } \quad \eta \in \mathcal{V}^{*}, \quad \text { a.e. } \quad t \in(0, T)
$$

We show by using the Banach contraction principle that the operator $\Lambda$ has a unique fixed point $\eta^{*} \in \mathcal{V}^{*}$. Then $u_{\eta^{*}}$ is a solution to Problem 3.1, which concludes the existence part of the theorem. The uniqueness part follows from the uniqueness of the fixed point of $\Lambda$. Namely, let $u \in \mathcal{V}$ be a solution to Problem 3.1 and define the element $\eta \in \mathcal{V}^{*}$ by $\eta(t)=\mathcal{S} u(t)$ for a.e. $t \in(0, T)$. It follows that $u$ is a solution to the problem (6) and, by the uniqueness of solutions to (6), we obtain $u=u_{\eta}$. This implies $\wedge \eta=\mathcal{S} u_{\eta}=\mathcal{S} u=\eta$ and by the uniqueness of the fixed point of $\wedge$ we have $\eta=\eta^{*}$, so $u=u_{\eta^{*}}$, which completes the proof. 


\section{Quasistatic hemivariational inequalities}

Let $V$ and $Z$ be reflexive, separable Banach spaces, and let $H$ be a Hilbert space. Suppose that $V \subset Z \subset H \approx H^{*} \subset$ $Z^{*} \subset V^{*}$, where $H^{*}, Z^{*}$ and $V^{*}$ denote dual spaces to $H, Z$ and $V$, respectively, all embeddings are continuous and $V$ is compactly embedded in $Z$. We denote by $\langle\cdot, \cdot\rangle$ the duality of $V$ and $V^{*}$, and the pairing between $Z$ and $Z^{*}$ as well. Given a fixed number $0<T<\infty$ and $2 \leq p<\infty$, we introduce the following spaces: $\mathcal{V}=L^{p}(0, T ; V), Z=L^{p}(0, T ; Z)$, $\widehat{\mathcal{H}}=L^{2}(0, T ; H), Z^{*}=L^{q}\left(0, T ; Z^{*}\right), \mathcal{V}^{*}=L^{q}\left(0, T ; V^{*}\right)$ with $1 / p+1 / q=1$ and $\mathcal{W}=\left\{w \in \mathcal{V}: w^{\prime} \in \mathcal{V}^{*}\right\}$, where the time derivative is understood in the sense of vector-valued distributions. The latter is a separable, reflexive Banach space with the norm $\|w\|_{\mathcal{W}}=\|w\|_{\mathcal{V}}+\left\|w^{\prime}\right\|_{\mathcal{V}^{*}}$. We have $\mathcal{W} \subset \mathcal{V} \subset Z \subset \widehat{\mathcal{H}} \subset Z^{*} \subset \mathcal{V}^{*}$ with continuous embeddings. It is well known, cf. e.g. [3, Proposition 8.4.14], that the space $\mathcal{W}$ is embedded continuously in $C(0, T ; H)$ (the space of continuous functions on $[0, T]$ with values in $H)$, i.e. every element of $\mathcal{W}$, after a possible modification on a set of measure zero, has a unique continuous representative in $C(0, T ; H)$. Moreover, since $V$ is embedded compactly in $Z$, then so is $\mathcal{W}$ in $Z$, cf. [3, Theorem 8.4.13].

Consider the following evolutionary inclusion of the form:

$$
\left\{\begin{array}{l}
A(t) u^{\prime}(t)+B u(t)+M^{*} \partial J(t, M u(t)) \ni f(t) \quad \text { for a.e. } \quad t \in(0, T), \\
u(0)=u_{0}
\end{array}\right.
$$

We remark that, by the definition of the Clarke subdifferential, problem (7) is equivalent to the following inequality:

$$
\left\{\begin{array}{l}
\left\langle A(t) u^{\prime}(t)+B u(t)-f(t), v\right\rangle+J^{0}(t, M u(t) ; M v) \geq 0 \quad \text { for all } \quad v \in V, \quad \text { a.e. } \quad t \in(0, T) \\
u(0)=u_{0},
\end{array}\right.
$$

where $J^{0}$ stands for the generalized directional derivative of $J(t, \cdot)$. For this reason problem (7) is called a hemivariational inequality.

\section{Definition 4.1.}

A function $u \in L^{\infty}(0, T ; V)$ is called a solution to (7) if and only if $u^{\prime} \in \mathcal{V}$ and there exists $\eta \in Z^{*}$ such that

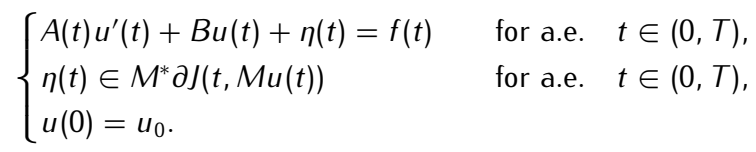

We note that if $u$ is a solution to (7), then $u \in L^{\infty}(0, T ; V) \subset \mathcal{V}$, i.e. $u \in W^{1, p}(0, T ; V)$. Since $W^{1, p}(0, T ; V) \subset C(0, T ; V)$ continuously, the initial condition $u(0)$ has a meaning in $V$.

In addition to our notation, let $X$ be a Banach space. We assume the following hypotheses.

$\mathrm{H}(A) \quad A \in L^{\infty}\left(0, T ; \mathcal{L}\left(V, V^{*}\right)\right)$ is an operator such that $A(t)$ is coercive, i.e. there is a constant $\alpha>0$ such that for a.e. $t \in(0, T),\langle A(t) v, v\rangle \geq \alpha\|v\|_{v}^{p}$ for all $v \in V$.

$\mathrm{H}(B) \quad B \in \mathcal{L}\left(V, V^{*}\right)$ is monotone (nonnegative) and symmetric.

$\mathrm{H}(J) \quad J:(0, T) \times X \rightarrow \mathbb{R}$ is a function such that

(i) $J(\cdot, x)$ is measurable on $(0, T)$ for all $x \in X$;

(ii) $J(t, \cdot)$ is locally Lipschitz on $X$ for a.e. $t \in(0, T)$;

(iii) $\|\partial J(t, x)\|_{X^{*}} \leq c\left(1+\|x\|_{X}^{2 / q}\right)$ for all $x \in X$, a.e. $t \in(0, T)$ with $c>0$.

$\mathrm{H}(M) \quad M \in \mathcal{L}(Z, X)$. 
$\mathrm{H}_{0} \quad f \in \mathcal{V}^{*}, u_{0} \in V, u_{1} \in H$.

$\mathrm{H}_{1} \quad$ If $p=2$, then $\alpha>2 c T c_{e}^{2}\|M\| \max \{1,\|M\|\}$, where $c_{e}>0$ is an embedding constant of $V$ into $Z$ and $\|M\|=$ $\|M\|_{\mathcal{L}(Z, X)}$.

The following is the main existence result on the evolution inclusion (7).

Theorem 4.2.

Under hypotheses $\mathrm{H}(A), \mathrm{H}(B), \mathrm{H}(J), \mathrm{H}(M), f \in \mathcal{V}^{*}, u_{0} \in V$ and $\mathrm{H}_{1}$, the inclusion (7) admits at least one solution.

The proof of Theorem 4.2 is based on the so-called vanishing acceleration method which we describe below. To this end, we consider an evolution second order inclusion of the form

$$
\left\{\begin{array}{l}
\varepsilon u^{\prime \prime}(t)+A(t) u^{\prime}(t)+B u(t)+M^{*} \partial J(t, M u(t)) \ni f(t) \quad \text { for a.e. } \quad t \in(0, T) \\
u(0)=u_{0}, \quad \sqrt{\varepsilon} u^{\prime}(0)=u_{1}
\end{array}\right.
$$

where $\varepsilon>0$. For $\varepsilon$ fixed, we write for simplicity $u$ for the solution $u_{\varepsilon}$ of (8).

\section{Definition 4.3.}

A function $u \in \mathcal{V}$ is called a solution to (8) if and only if $u^{\prime} \in \mathcal{W}$ and there exists $\eta \in Z^{*}$ such that

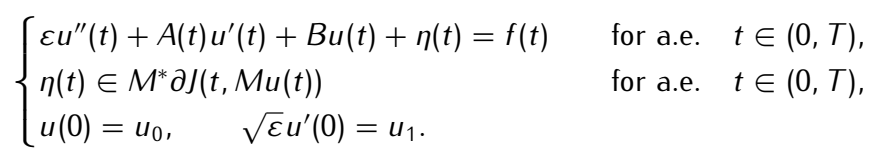

Similarly, as before, we note that if $u$ is a solution to (8), then $u \in W^{1, p}(0, T ; V)$. Since the embeddings $W^{1, p}(0, T ; V) \subset$ $C(0, T ; V)$ and $\mathcal{W} \subset C(0, T ; H)$ are continuous, the initial conditions $u(0)$ and $u^{\prime}(0)$ have a meaning in $V$ and $H$, respectively.

First, we comment on the existence result for (8). The important step is to derive the following uniform estimate.

\section{Lemma 4.4}

Let $\varepsilon>0$ be fixed. Assume hypotheses $\mathrm{H}(A), \mathrm{H}(B), \mathrm{H}(J), \mathrm{H}(M)$ and $\mathrm{H}_{0}$, and let $u$ be a solution to (8). If $p>2$, then there exists a constant $C>0$ independent of $\varepsilon$ such that

$$
\|u\|_{C(0, T ; V)}+\left\|u^{\prime}\right\|_{\mathcal{V}}+\sqrt{\varepsilon}\left\|u^{\prime}\right\|_{L^{\infty}(0, T ; H)}+\varepsilon\left\|u^{\prime \prime}\right\|_{\mathcal{V}^{*}} \leq C\left(1+\left\|u_{0}\right\|_{V^{2 / q}}^{2 / \|}\left\|u_{1}\right\|_{H}^{2 / q}+\|f\|_{\mathcal{V}^{*}}^{2 / q}\right)
$$

Moreover, this estimate still holds for $p=2$ provided $\mathrm{H}_{1}$ is satisfied.

From [13, Theorem 8], we obtain the following result.

Theorem 4.5.

If hypotheses $\mathrm{H}(A), \mathrm{H}(B), \mathrm{H}(J), \mathrm{H}(M), \mathrm{H}_{0}$ and $\mathrm{H}_{1}$ hold, then for every fixed $\varepsilon>0$ the problem (8) admits at least one solution.

The following is the existence result for the operator inclusion (7). 
Theorem 4.6.

Let the hypotheses $\mathrm{H}(A), \mathrm{H}(B), \mathrm{H}(J), \mathrm{H}(M), \mathrm{H}_{0}$ and $\mathrm{H}_{1}$ hold. For every $\varepsilon$ fixed, let $u_{\varepsilon}$ be a solution to (8) given by Theorem 4.5. Then there exists u fulfilling $u \in L^{\infty}(0, T ; V), u^{\prime} \in \mathcal{W}$, such that the following convergences hold:

$$
\begin{aligned}
u_{\varepsilon} \rightarrow u & \text { weakly* in } L^{\infty}(0, T ; V), \quad u_{\varepsilon}^{\prime} \rightarrow u^{\prime} \quad \text { weakly in } \mathcal{V}, \\
\sqrt{\varepsilon} u_{\varepsilon}^{\prime} \rightarrow 0 & \text { weakly* in } L^{\infty}(0, T ; H), \quad \varepsilon u_{\varepsilon}^{\prime \prime} \rightarrow 0 \quad \text { weakly in } \mathcal{V}^{*},
\end{aligned}
$$

as $\varepsilon \rightarrow 0$. Moreover, the limit function $u$ is a solution to the problem (7).

The study of inclusions of type (7) is motivated by several contact problems of solid mechanics. It is well known that the dynamic equations of motion, representing momentum conservation, that govern the evolution of the state of the body, are of the form $u_{i}^{\prime \prime}-\sigma_{i j, j}=f_{i}$, where $u$ is a displacement, $\sigma$ is the stress tensor and $f$ is the density (per unit volume) of applied forces. In many cases we are interested in situations in which the system configuration and the external forces and tractions evolve slowly in time in such a way that the accelerations in the system are rather small and negligible, so that the inertial (the second order time derivative) terms can be neglected. In such a way, we obtain the quasistatic approximation (equilibrium equations) for the equations of motion, $-\operatorname{Div} \sigma=f$, where Div is the divergence operator. In this approximation, at each time instant the system is in equilibrium, and the external forces are balanced by the internal stresses. Rigorous mathematical treatment of quasistatic problems is recent. The rapid growth of the theory of quasistatic contact models can be seen from several monographs and many papers dedicated to such phenomena, cf. Duvaut and Lions [4], Han and Sofonea [6], Shillor et al. [19] and references therein. When we assume that the process is slow and the accelerations are negligible, mathematically it means that the system changes character, from being of a hyperbolic type to an elliptic or a parabolic type. To our knowledge quasistatic hemivariational inequalities have been studied in the literature only recently and a recent existence result for such problems is provided in Migórski and Ochal [13]. We mention that quasistatic problems modeled by variational inequalities with strongly monotone and Lipschitz operators were considered in $[6,19]$. We also point out that the result of Theorem 4.6 can be applicable to several important and challenging quasistatic models of contact phenomena. Finally, we remark that the question of uniqueness of solutions to both dynamical and quasistatic hemivariational inequality is left open. It would be also interesting to extend the result of Theorem 4.6 to a class of problems with nonlinear operators $A(t)$ and $B$.

\section{History-dependent hemivariational inequality}

In this section we provide a result on existence and uniqueness of a solution to a class of hemivariational inequalities associated with Problem 3.1. We adopt the notation of Section 3. The problem under consideration reads as follows.

\section{Problem 5.1.}

Find $u \in \mathcal{V}$ such that

$$
\langle A(t, u(t)), v\rangle_{V^{*} \times V}+\langle\mathcal{S} u(t), v\rangle_{V^{*} \times V}+\int_{\Gamma} j^{0}(t, \gamma u(t) ; \gamma v) d \Gamma \geq\langle f(t), v\rangle_{V^{*} \times V}
$$

for all $v \in V$ and a.e. $t \in(0, T)$.

In the study of the hemivariational inequality (9), in addition to the previous assumptions, we need the following hypothesis.

V. $j: \Gamma \times(0, T) \times \mathbb{R}^{s} \rightarrow \mathbb{R}$ is such that

(V.a) $j(\cdot, \cdot, \xi)$ is measurable on $\Gamma \times(0, T)$ for all $\xi \in \mathbb{R}^{s}$ and $j(\cdot, \cdot, 0) \in L^{1}(\Gamma \times(0, T))$;

(V.b) $j(x, t, \cdot)$ is locally Lipschitz on $\mathbb{R}^{s}$ for a.e. $(x, t) \in \Gamma \times(0, T)$; 
(V.c) $\|\partial j(x, t, \xi)\|_{\mathbb{R}^{s}} \leq \bar{c}_{0}+\bar{c}_{1}\|\xi\|_{\mathbb{R}^{s}}$ for a.e. $(x, t) \in \Gamma \times(0, T)$, all $\xi \in \mathbb{R}^{s}$ with $\bar{c}_{0}, \bar{c}_{1} \geq 0$;

(V.d) $\left(\zeta_{1}-\zeta_{2}\right) \cdot\left(\xi_{1}-\xi_{2}\right) \geq-m_{2}\left\|\xi_{1}-\xi_{2}\right\|_{\mathbb{R}^{s}}^{2}$ for all $\zeta_{i}, \xi_{i} \in \mathbb{R}^{s}, \zeta_{i} \in \partial j\left(x, t, \xi_{i}\right), i=1,2$, a.e. $(x, t) \in \Gamma \times(0, T)$ with $m_{2} \geq 0$

(V.e) $j^{0}(x, t, \xi ;-\xi) \leq \bar{d}_{0}\left(1+\|\xi\|_{\mathbb{R}^{s}}\right)$ for a.e. $(x, t) \in \Gamma \times(0, T)$, all $\xi \in \mathbb{R}^{s}$ with $\bar{d}_{0} \geq 0$.

Note that in the condition (V.d) the dot denotes the inner product in $\mathbb{R}^{s}$. From Theorem 3.4, we deduce the following existence and uniqueness result for the hemivariational inequality (9). The details on its proof can be found in [14].

Theorem 5.2.

Assume that I and II hold and $f \in \mathcal{V}^{*}$. If one of the following hypotheses:

(i) (V.a)-(V.d) and $m_{1}>\max \left\{\sqrt{3} \bar{c}_{1}, m_{2}\right\} c_{e}^{2}\|\gamma\|^{2}$,

(ii) $\vee$ and $m_{1}>m_{2} c_{e}^{2}\|\gamma\|^{2}$

is satisfied, then Problem 5.1 has a solution $u \in \mathcal{V}$. If, in addition, the regularity condition:

either $j(x, t, \cdot)$ or $-j(x, t, \cdot) \quad$ is regular on $\mathbb{R}^{s}$ for a.e. $(x, t) \in \Gamma \times(0, T)$

holds, then the solution of Problem 5.1 is unique.

\section{Quasi-static frictional contact problems}

A large number of quasistatic contact problems with elastic or viscoelastic materials leads to a hemivariational inequality of the form (9) in which the unknown is either the velocity or the displacement field. In both cases the abstract results of Sections 3, 4 and 5 work and can be used to provide the unique weak solvability of the corresponding contact problems. In this section we illustrate the use of these results in the study of two contact problems.

The physical setting of the first contact problem is as follows. A viscoelastic body occupies a domain $\Omega$ of $\mathbb{R}^{d}, d=2,3$, with surface $\partial \Omega$ which is partitioned into three disjoint measurable parts $\Gamma_{D}, \Gamma_{N}$ and $\Gamma_{C}$ such that $m\left(\Gamma_{D}\right)>0$. We are interested in the evolution process of the mechanical state of the body in the bounded interval of time $[0, T]$, where $T>0$. The body is clamped on $\Gamma_{D}$ and so the displacement field vanishes there. Surface tractions of density $\boldsymbol{f}_{N}$ act on $\Gamma_{N}$ and volume forces of density $f_{0}$ act in $\Omega$. We assume that the forces and tractions change slowly in time so that the acceleration of the system is negligible and, therefore, the process is quasistatic. Moreover, the body is in contact with an obstacle on $\Gamma_{C}$, the so-called foundation. The contact is frictional and is modeled with subdifferential boundary conditions.

We use the notation $\boldsymbol{v}=\left(v_{i}\right)$ for the outward unit normal at $\partial \Omega$. We denote by $\boldsymbol{u}=\left(u_{i}\right), \boldsymbol{\sigma}=\left(\sigma_{i j}\right)$, and $\boldsymbol{\varepsilon}(\boldsymbol{u})=\left(\varepsilon_{i j}(\boldsymbol{u})\right)$ the displacement vector, the stress tensor, and the linearized strain tensor, respectively. We recall that the components of the linearized strain tensor $\varepsilon(\boldsymbol{u})$ are given by $\varepsilon_{i j}(\boldsymbol{u})=\left(u_{i, j}+u_{j, i}\right) / 2$. We denote by $\mathbb{S}^{d}$ the space of second order symmetric tensors on $\mathbb{R}^{d}$ or, equivalently, the space of symmetric matrices of order $d$. The canonical inner products and the corresponding norms on $\mathbb{R}^{d}$ and $\mathbb{S}^{d}$ are given by

$$
\begin{aligned}
& \boldsymbol{u} \cdot \boldsymbol{v}=u_{i} v_{i}, \quad\|\boldsymbol{v}\|_{\mathbb{R}^{d}}=(\boldsymbol{v} \cdot \boldsymbol{v})^{1 / 2} \quad \text { for all } \quad \boldsymbol{u}=\left(u_{i}\right), \quad \boldsymbol{v}=\left(v_{i}\right) \in \mathbb{R}^{d}, \\
& \boldsymbol{\sigma}: \boldsymbol{\tau}=\sigma_{i j} \tau_{i j}, \quad\|\boldsymbol{\tau}\|_{\mathbb{S}^{d}}=(\boldsymbol{\tau}: \boldsymbol{\tau})^{1 / 2} \quad \text { for all } \quad \boldsymbol{\sigma}=\left(\sigma_{i j}\right), \quad \boldsymbol{\tau}=\left(\tau_{i j}\right) \in \mathbb{S}^{d} \text {, }
\end{aligned}
$$

respectively.

With these preliminaries, the classical formulation of the quasistatic contact problem we consider in this section is the following. 
Problem 6.1.

Find the displacement field $\boldsymbol{u}: \Omega \times[0, T] \rightarrow \mathbb{R}^{d}$ and the stress field $\boldsymbol{\sigma}: \Omega \times[0, T] \rightarrow \mathbb{S}^{d}$ such that, for all $t \in(0, T)$,

$$
\begin{aligned}
\boldsymbol{\sigma}(t)=\mathcal{A}\left(t, \boldsymbol{\varepsilon}\left(\boldsymbol{u}^{\prime}(t)\right)\right)+\mathcal{B}(t, \boldsymbol{\varepsilon}(\boldsymbol{u}(t))) & \text { in } \Omega, \\
\operatorname{Div} \boldsymbol{\sigma}(t)+\boldsymbol{f}_{0}(t)=0 & \text { in } \Omega, \\
\boldsymbol{u}(t)=0 & \text { on } \Gamma_{D}, \\
\boldsymbol{\sigma}(t) \boldsymbol{v}=\boldsymbol{f}_{N}(t) & \text { on } \Gamma_{N}, \\
-\sigma_{v}(t) \in \partial j_{v}\left(t, u_{v}^{\prime}(t)\right) & \text { on } \Gamma_{C}, \\
-\boldsymbol{\sigma}_{\tau}(t) \in \partial j_{\tau}\left(t, \boldsymbol{u}_{\tau}^{\prime}(t)\right) & \text { on } \Gamma_{C}, \\
\boldsymbol{u}(0)=\boldsymbol{u}_{0} & \text { in } \Omega .
\end{aligned}
$$

We present a short description of the equations and conditions in Problem 6.1 and we refer the reader to $[6,15,19]$ for more details and mechanical interpretation.

The equation (10a) represents the viscoelastic constitutive law in which $\mathcal{A}$ and $\mathcal{B}$ are given nonlinear operators, called the viscosity operator and elasticity operator, respectively. The explicit dependence of the viscosity and elasticity operators $\mathcal{A}$ and $\mathcal{B}$ with respect to the time variable allows to model situations when the properties of the material depend on the temperature, which plays the role of a parameter, i.e. its evolution in time is prescribed. Equality (10b) is the equilibrium equation, where Div represents the divergence operator, i.e. Div $\boldsymbol{\sigma}=\left(\sigma_{i j, j}\right)$. Conditions (10c) and (10d) are the displacement and traction boundary conditions, respectively, and $(10 \mathrm{~g})$ is the initial condition in which the function $\boldsymbol{u}_{0}$ denotes the initial displacement field.

Conditions (10e) and (10f) represent the frictional contact conditions in which $j_{v}$ and $j_{\tau}$ are given functions. The subscripts $v$ and $\tau$ for $\boldsymbol{\sigma}$ and $\boldsymbol{u}^{\prime}$ indicate normal and tangential components of tensors and vectors. The symbol $\partial j$ denotes the Clarke subdifferential of $j$ with respect to the last variable. Concrete examples of frictional models which lead to subdifferential boundary conditions of the form (10e), (10f) with the functions $j_{v}$ and $j_{\tau}$ satisfying assumptions VIII and IX below can be found in $[12,15]$. Here, we restrict ourselves to remark that these examples include the viscous contact and the contact with nonmonotone normal damped response, associated to a nonmonotone friction law, to Tresca's friction law or to a power-law friction. Our results below are valid for the corresponding quasistatic frictional contact problems.

In the study of the contact problem (10a)-(10g) we use standard notation $Q=\Omega \times(0, T), \Sigma_{D}=\Gamma_{D} \times(0, T), \Sigma_{N}=$ $\Gamma_{N} \times(0, T)$ and $\Sigma_{C}=\Gamma_{C} \times(0, T)$. For all $v \in H^{1}\left(\Omega ; \mathbb{R}^{d}\right)$ we still denote by $v$ the trace of $v$ on $\Gamma$ and we use the notation $v_{v}$ and $\boldsymbol{v}_{\tau}$ for the normal and tangential components of $\boldsymbol{v}$ on $\partial \Omega$ given by $v_{v}=\boldsymbol{v} \cdot \boldsymbol{v}$ and $\boldsymbol{v}_{\tau}=\boldsymbol{v}-\boldsymbol{v}_{v} \boldsymbol{v}$. We recall that the normal and tangential components of the stress field $\boldsymbol{\sigma}$ on the boundary are defined by $\sigma_{v}=(\boldsymbol{\sigma} \boldsymbol{v}) \cdot \boldsymbol{v}$ and $\boldsymbol{\sigma}_{\tau}=\boldsymbol{\sigma} \boldsymbol{v}-\sigma_{v} \boldsymbol{v}$. Next, we introduce the spaces $V$ and $\mathcal{H}$, defined by $V=\left\{\boldsymbol{v}=\left(v_{i}\right) \in H^{1}\left(\Omega ; \mathbb{R}^{d}\right): \boldsymbol{v}=\mathbf{0}\right.$ a.e. on $\left.\Gamma_{D}\right\}$ and $\mathcal{H}=L^{2}\left(\Omega ; \mathbb{S}^{d}\right)$.

We assume that the viscosity operator $\mathcal{A}$ and the elasticity operator $\mathcal{B}$ satisfy

VI. $\mathcal{A}: Q \times \mathbb{S}^{d} \rightarrow \mathbb{S}^{d}$ is such that

(VI.a) $\mathcal{A}(\cdot, \cdot, \varepsilon)$ is measurable on $Q$ for all $\varepsilon \in \mathbb{S}^{d}$;

(VI.b) $\mathcal{A}(x, t, \cdot)$ is continuous on $\mathbb{S}^{d}$ for a.e. $(x, t) \in Q$;

(VI.C) $\left(\mathcal{A}\left(x, t, \varepsilon_{1}\right)-\mathcal{A}\left(x, t, \varepsilon_{2}\right)\right):\left(\varepsilon_{1}-\varepsilon_{2}\right) \geq m_{\mathcal{A}}\left\|\varepsilon_{1}-\varepsilon_{2}\right\|_{\mathbb{S}^{d}}^{2}$ for all $\varepsilon_{1}, \varepsilon_{2} \in \mathbb{S}^{d}$, a.e. $(x, t) \in Q$ with $m_{\mathcal{A}}>0$;

(VI.d) $\|\mathcal{A}(x, t, \varepsilon)\|_{\mathbb{S}^{d}} \leq \bar{a}_{0}(x, t)+\bar{a}_{1}\|\varepsilon\|_{\mathbb{S}^{d}}$ for all $\varepsilon \in \mathbb{S}^{d}$, a.e. $(x, t) \in Q$ with $\bar{a}_{0} \in L^{2}(Q), \bar{a}_{0} \geq 0$ and $\bar{a}_{1}>0$;

(VI.e) $\mathcal{A}(x, t, 0)=0$ for a.e. $(x, t) \in Q$.

VII. $\mathcal{B}: Q \times \mathbb{S}^{d} \rightarrow \mathbb{S}^{d}$ is such that

(VII.a) $\mathcal{B}(\cdot, \cdot, \varepsilon)$ is measurable on $Q$ for all $\varepsilon \in \mathbb{S}^{d}$;

(VII.b) $\left\|\mathcal{B}\left(x, t, \varepsilon_{1}\right)-\mathcal{B}\left(x, t, \varepsilon_{2}\right)\right\|_{\mathbb{S}^{d}} \leq L_{\mathcal{B}}\left\|\varepsilon_{1}-\varepsilon_{2}\right\|_{\mathbb{S}^{d}}$ for all $\varepsilon_{1}, \varepsilon_{2} \in \mathbb{S}^{d}$, a.e. $(x, t) \in Q$ with $L_{\mathcal{B}}>0$;

(VII.c) $\mathcal{B}(\cdot, \cdot, 0) \in L^{2}\left(Q ; \mathbb{S}^{d}\right)$. 
The contact potentials $j_{v}$ and $j_{\tau}$ satisfy the following hypotheses.

VIII. $j_{v}: \Sigma_{C} \times \mathbb{R} \rightarrow \mathbb{R}$ is such that

(VIII.a) $j_{v}(\cdot, \cdot, r)$ is measurable on $\Sigma_{C}$ for all $r \in \mathbb{R}$ and $j_{v}(\cdot, \cdot, 0) \in L^{1}\left(\Sigma_{C}\right)$;

(VIII.b) $j_{v}(x, t, \cdot)$ is locally Lipschitz on $\mathbb{R}$ for a.e. $(x, t) \in \Sigma_{C}$;

(VIII.c) $\left|\partial j_{v}(x, t, r)\right| \leq c_{0 v}+c_{1 v}|r|$ for all $r \in \mathbb{R}$, a.e. $(x, t) \in \Sigma_{C}$ with $c_{0 v}, c_{1 v} \geq 0$;

(VIII.d) $\left(\zeta_{1}-\zeta_{2}\right)\left(r_{1}-r_{2}\right) \geq-m_{v}\left|r_{1}-r_{2}\right|^{2}$ for all $\zeta_{i} \in \partial j_{v}\left(x, t, r_{i}\right), r_{i} \in \mathbb{R}, i=1,2$, a.e. $(x, t) \in \Sigma_{C}$ with $m_{v} \geq 0$;

(VIII.e) $j_{v}^{0}(x, t, r ;-r) \leq d_{v}(1+|r|)$ for all $r \in \mathbb{R}$, a.e. $(x, t) \in \Sigma_{C}$ with $d_{v} \geq 0$.

IX. $j_{\tau}: \Sigma_{C} \times \mathbb{R}^{d} \rightarrow \mathbb{R}$ is such that

(IX.a) $j_{\tau}(\cdot, \cdot, \xi)$ is measurable on $\Sigma_{C}$ for all $\xi \in \mathbb{R}^{d}$ and $j_{\tau}(\cdot, \cdot, 0) \in L^{1}\left(\Sigma_{C}\right)$;

(IX.b) $j_{\tau}(x, t, \cdot)$ is locally Lipschitz on $\mathbb{R}^{d}$ for a.e. $(x, t) \in \Sigma_{C}$;

(IX.c) $\left\|\partial j_{\tau}(x, t, \xi)\right\|_{\mathbb{R}^{d}} \leq c_{0 \tau}+c_{1 \tau}\|\xi\|_{\mathbb{R}^{d}}$ for all $\xi \in \mathbb{R}^{d}$, a.e. $(x, t) \in \Sigma_{C}$ with $c_{0 \tau}, c_{1 \tau} \geq 0$;

(IX.d) $\left(\boldsymbol{\zeta}_{1}-\boldsymbol{\zeta}_{2}\right) \cdot\left(\boldsymbol{\xi}_{1}-\boldsymbol{\xi}_{2}\right) \geq-m_{\tau}\left\|\boldsymbol{\xi}_{1}-\boldsymbol{\xi}_{2}\right\|_{\mathbb{R}^{d}}^{2}$ for all $\boldsymbol{\zeta}_{i} \in \partial j_{\tau}\left(x, t, \boldsymbol{\xi}_{i}\right), \boldsymbol{\xi}_{i} \in \mathbb{R}^{d}, i=1,2$, a.e. $(x, t) \in \Sigma_{C}$ with $m_{\tau} \geq 0$;

(IX.e) $j_{\tau}^{0}(x, t, \xi ;-\xi) \leq d_{\tau}\left(1+\|\xi\|_{\mathbb{R}^{d}}\right)$ for all $\xi \in \mathbb{R}^{d}$, a.e. $(x, t) \in \Sigma_{C}$ with $d_{\tau} \geq 0$.

The volume force and traction densities satisfy

$$
\boldsymbol{f}_{0} \in L^{2}\left(0, T ; L^{2}\left(\Omega ; \mathbb{R}^{d}\right)\right), \quad \boldsymbol{f}_{N} \in L^{2}\left(0, T ; L^{2}\left(\Gamma_{N} ; \mathbb{R}^{d}\right)\right)
$$

and, finally, the initial displacement is such that

$$
\boldsymbol{u}_{0} \in V .
$$

We turn now to the variational formulation of Problem 6.1. Suppose that $(\boldsymbol{u}, \boldsymbol{\sigma})$ is a couple of sufficiently smooth functions which solve (10a)-(10g). Let $v \in V$. Then, using (10b), we have

$$
(\boldsymbol{\sigma}(t), \varepsilon(\boldsymbol{v}))_{\mathcal{H}}=\left(f_{0}(t), v\right)_{L^{2}\left(\Omega ; \mathbb{R}^{d}\right)}+\int_{\partial \Omega} \boldsymbol{\sigma}(t) \boldsymbol{v} \cdot \boldsymbol{v} d \Gamma \quad \text { for a.e. } \quad t \in(0, T) .
$$

We take into account the boundary conditions (10c) and (10d) to see that

$$
\int_{\partial \Omega} \boldsymbol{\sigma}(t) \boldsymbol{v} \cdot \boldsymbol{v} d \Gamma=\int_{\Gamma_{N}} \boldsymbol{f}_{N}(t) \cdot \boldsymbol{v} d \Gamma+\int_{\Gamma_{C}}\left(\sigma_{v}(t) v_{v}+\boldsymbol{\sigma}_{\tau}(t) \cdot \boldsymbol{v}_{\tau}\right) d \Gamma \quad \text { for a.e. } \quad t \in(0, T) .
$$

On the other hand, from the definition of the Clarke subdifferential, (10e) and (10f), we have

$$
-\sigma_{v}(t) v_{v} \leq j_{v}^{0}\left(t, u_{v}^{\prime}(t) ; v_{v}\right), \quad-\sigma_{\tau}(t) \cdot v_{\tau} \leq j_{\tau}^{0}\left(t, u_{\tau}^{\prime}(t) ; v_{\tau}\right) \quad \text { on } \quad \Sigma_{C} \text {, }
$$

which imply that

$$
\int_{\Gamma_{C}}\left(\sigma_{v}(t) v_{v}+\sigma_{\tau}(t) \cdot v_{\tau}\right) d \Gamma \geq-\int_{\Gamma_{C}}\left(j_{v}^{0}\left(t, u_{v}^{\prime}(t) ; v_{v}\right)+j_{\tau}^{0}\left(t, u_{\tau}^{\prime}(t) ; v_{\tau}\right)\right) d \Gamma
$$

for a.e. $t \in(0, T)$. Consider the function $f:(0, T) \rightarrow V^{*}$ given by

$$
\langle\boldsymbol{f}(t), \boldsymbol{v}\rangle_{V^{*} \times V}=\left(\boldsymbol{f}_{0}(t), \boldsymbol{v}\right)_{L^{2}\left(\Omega ; \mathbb{R}^{d}\right)}+\left(f_{N}(t), v\right)_{L^{2}\left(\Gamma_{N} ; \mathbb{R}^{d}\right)}
$$

for all $v \in V$ and a.e. $t \in(0, T)$. We combine (13)-(16) to obtain

$$
(\boldsymbol{\sigma}(t), \boldsymbol{\varepsilon}(\boldsymbol{v}))_{\mathcal{H}}+\int_{\Gamma_{C}}\left(j_{v}^{0}\left(t, u_{v}^{\prime}(t) ; v_{v}\right)+j_{\tau}^{0}\left(t, \boldsymbol{u}_{\tau}^{\prime}(t) ; v_{\tau}\right)\right) d \Gamma \geq\langle\boldsymbol{f}(t), v\rangle_{V^{*} \times V}
$$


and, using the constitutive law (10a), it follows

$$
\left(\mathcal{A}\left(t, \boldsymbol{\varepsilon}\left(\boldsymbol{u}^{\prime}(t)\right)\right), \boldsymbol{\varepsilon}(\boldsymbol{v})\right)_{\mathcal{H}}+(\mathcal{B}(t, \boldsymbol{\varepsilon}(\boldsymbol{u}(t))), \boldsymbol{\varepsilon}(\boldsymbol{v}))_{\mathcal{H}}+\int_{\Gamma_{C}}\left(j_{v}^{0}\left(t, u_{v}^{\prime}(t) ; v_{v}\right)+j_{\tau}^{0}\left(t, \boldsymbol{u}_{\tau}^{\prime}(t) ; \boldsymbol{v}_{\tau}\right)\right) d \Gamma \geq\langle\boldsymbol{f}(t), \boldsymbol{v}\rangle_{V^{*} \times V}
$$

for all $v \in V$ and a.e. $t \in(0, T)$. Let $w=\boldsymbol{u}^{\prime}$ denote the velocity field. Then, by using the initial condition (10g), it follows that

$$
\boldsymbol{u}(t)=\int_{0}^{t} w(s) d s+\boldsymbol{u}_{0} \quad \text { for all } \quad t \in[0, T] .
$$

Therefore, (17) and (18) lead to the following variational formulation of problem (10a)-(10g), in terms of velocity.

\section{Problem 6.2.}

Find a velocity field $w \in \mathcal{V}$ such that

$$
(\mathcal{A}(t, \boldsymbol{\varepsilon}(\boldsymbol{w}(t))), \boldsymbol{\varepsilon}(\boldsymbol{v}))_{\mathcal{H}}+\left(\mathcal{B}\left(t, \boldsymbol{\varepsilon}\left(\int_{0}^{t} w(s) d s+\boldsymbol{u}_{0}\right)\right), \boldsymbol{\varepsilon}(\boldsymbol{v})\right)_{\mathcal{H}}+\int_{\Gamma_{C}}\left(j_{v}^{0}\left(t, w_{v}(t) ; v_{v}\right)+j_{\tau}^{0}\left(t, \boldsymbol{w}_{\tau}(t) ; \boldsymbol{v}_{\tau}\right)\right) d \Gamma \geq\langle\boldsymbol{f}(t), \boldsymbol{v}\rangle_{V^{*} \times V}
$$

for all $v \in V$ and a.e. $t \in(0, T)$.

The hemivariational inequality in Problem 6.2 is of the form of the inequality in Problem 5.1. From Theorem 5.2, we obtain the following result on Problem 6.2.

\section{Theorem 6.3.}

Assume that (VI.a), VII, (11) and (12) hold. If one of the following hypotheses:

(i) (VIII.a)-(VIII.d), (IX.a)-(IX.d) and $m_{1}>\max \left\{\sqrt{3}\left(c_{1 v}+c_{1 \tau}\right), m_{v}, m_{\tau}\right\} c_{e}^{2}\|\gamma\|^{2}$,

(ii) VIII, IX and $m_{1}>\max \left\{m_{v}, m_{\tau}\right\} c_{e}^{2}\|\gamma\|^{2}$

is satisfied, then Problem 6.2 has at least one solution. If, in addition,

$$
\text { either } j_{v}(x, t, \cdot), j_{\tau}(x, t, \cdot) \quad \text { are regular or }-j_{v}(x, t, \cdot),-j_{\tau}(x, t, \cdot) \text { are regular for a.e. }(x, t) \in \Sigma_{C} \text {, }
$$

then the solution of Problem 6.2 is unique.

Let $\boldsymbol{w}$ be a solution of Problem 6.2 and denote by $\boldsymbol{u}$ and $\boldsymbol{\sigma}$ the functions defined by (18) and (10a). Then, the couple $(\boldsymbol{u}, \boldsymbol{\sigma})$ is called a weak solution of the frictional contact problem $(10 \mathrm{a})-(10 \mathrm{~g})$. We conclude, under the hypotheses of Theorem 6.3, that the frictional contact problem $(10 \mathrm{a})-(10 \mathrm{~g})$ has at least one weak solution with the following regularity:

$$
\boldsymbol{u} \in W^{1,2}(0, T ; V) \quad \text { and } \quad \boldsymbol{\sigma} \in L^{2}(0, T ; \mathcal{H}) \quad \text { with } \quad \operatorname{Div} \boldsymbol{\sigma} \in L^{2}\left(0, T ; V^{*}\right)
$$

If, in addition, the regularity condition (19) holds, then the weak solution of Problem 6.1 is unique.

We now pass to the second problem of this section. We consider the quasistatic viscoelastic contact with nonmonotone normal compliance and friction. As in the first problem, we assume that the volume forces and surface tractions change slowly in time so that the acceleration in the system is negligible. We show that the quasistatic model can be formulated as a time dependent hemivariational inequality of the form (7) and the abstract result of Theorem 3.4 is applicable in this case. For the mechanical formulation of the process we use the notation introduced above. We set $Q=\Omega \times(0, T)$. Again we denote by $\boldsymbol{u}: Q \rightarrow \mathbb{R}^{d}$ the displacement field, by $\boldsymbol{\sigma}: Q \rightarrow \mathbb{S}^{d}$ the stress tensor and by $\boldsymbol{\varepsilon}(\boldsymbol{u})=\left\{\boldsymbol{\varepsilon}_{i j}(\boldsymbol{u})\right\}$, $\boldsymbol{\varepsilon}_{i j}(\boldsymbol{u})=\left(\partial_{j} \boldsymbol{u}_{i}+\partial_{i} \boldsymbol{u}_{j}\right) / 2$ the strain tensor. We assume a linear viscoelastic model with the constitutive law of the KelvinVoigt type

$$
\sigma_{i j}=a_{i j k l} \varepsilon_{k l}\left(u^{\prime}\right)+b_{i j k l} \varepsilon_{k l}(u) \quad \text { in } Q \text {, }
$$


where $\mathcal{C}(t)=\left\{a_{i j k l}(t)\right\}$ and $\mathcal{G}=\left\{b_{i j k l}\right\}, i, j, k, l=1, \ldots, d$, are the viscosity and the elasticity tensors, respectively. Concerning the contact conditions, we consider the following subdifferential relations $-\sigma_{v} \in \partial j_{v}\left(x, t, u_{v}\right)$ and $-\sigma_{\tau} \in$ $\partial j_{\tau}\left(x, t, \boldsymbol{u}_{\tau}\right)$. The functions $j_{v}: \Gamma_{C} \times(0, T) \times \mathbb{R} \rightarrow \mathbb{R}$ and $j_{\tau}: \Gamma_{C} \times(0, T) \times \mathbb{R}^{d} \rightarrow \mathbb{R}$ are locally Lipschitz in their last variables and $\partial j_{v}, \partial j_{\tau}$ denote their Clarke subdifferentials. The initial displacement is denoted by $\boldsymbol{u}_{0}$. The classical formulation of the mechanical problem is as follows. Find the displacement field $u: \Omega \times[0, T] \rightarrow \mathbb{R}^{d}$ and the stress field $\boldsymbol{\sigma}: \Omega \times[0, T] \rightarrow \mathbb{S}^{d}$ such that, for all $t \in(0, T)$,

$$
\begin{aligned}
\boldsymbol{\sigma}(t)=\mathcal{C}(t) \boldsymbol{\varepsilon}\left(\boldsymbol{u}^{\prime}(t)\right)+\mathcal{G} \boldsymbol{\varepsilon}(\boldsymbol{u}(t)) & \text { in } \Omega, \\
\operatorname{Div} \boldsymbol{\sigma}(t)+\boldsymbol{f}_{0}(t)=\mathbf{0} & \text { in } \Omega, \\
\boldsymbol{u}(t)=\mathbf{0} & \text { on } \Gamma_{D}, \\
\boldsymbol{\sigma}(t) \boldsymbol{v}=\boldsymbol{f}_{N}(t) & \text { on } \Gamma_{N}, \\
-\sigma_{v}(t) \in \partial j_{v}\left(t, u_{v}(t)\right) & \text { on } \Gamma_{C}, \\
-\boldsymbol{\sigma}_{\tau}(t) \in \partial j_{\tau}\left(t, \boldsymbol{u}_{\tau}(t)\right) & \text { on } \Gamma_{C}, \\
\boldsymbol{u}(0)=\boldsymbol{u}_{0} & \text { in } \Omega .
\end{aligned}
$$

For concrete examples of boundary conditions (20e) and (20f), we refer to [4, 6, 16-18].

In order to obtain a variational formulation of the problem $(20 \mathrm{a})-(20 \mathrm{~g})$ we need additional notation. Let $V=\left\{v=\left(v_{i}\right) \in\right.$ $H^{1}\left(\Omega ; \mathbb{R}^{d}\right): v=0$ a.e. on $\left.\Gamma_{D}\right\}, Z=H^{\delta}\left(\Omega ; \mathbb{R}^{d}\right)$ with a fixed $\delta \in(1 / 2,1), H=L^{2}\left(\Omega ; \mathbb{R}^{d}\right)$ and $X=L^{2}\left(\Gamma_{C} ; \mathbb{R}^{d}\right)$. On $V$ we consider the inner product and the corresponding norm given by $\langle\boldsymbol{u}, \boldsymbol{v}\rangle_{V}=\langle\varepsilon(\boldsymbol{u}), \boldsymbol{\varepsilon}(\boldsymbol{v})\rangle_{L^{2}\left(\Omega ; \mathbb{S}^{d}\right)},\|\boldsymbol{v}\|_{V}=\|\varepsilon(\boldsymbol{v})\|_{L^{2}\left(\Omega ; \mathbb{S}^{d}\right)}$ for $u, v \in V$. Since $m\left(\Gamma_{D}\right)>0$, the Korn inequality $\|v\|_{H^{1}\left(\Omega ; \mathbb{R}^{d}\right)} \leq c\|\varepsilon(v)\|_{L^{2}\left(\Omega ; \mathbb{S}^{d}\right)}$ for $v \in V$ with $c>0$ holds, and therefore the norms $\|\cdot\|_{H^{1}\left(\Omega ; \mathbb{R}^{d}\right)}$ and $\|\cdot\|_{v}$ are equivalent on $V$. Moreover, we put $M=\gamma \in \mathcal{L}(Z, X), \gamma$ is the trace operator, and, for simplicity, $p=2$.

We also assume that the viscosity and elasticity tensors have the usual properties of ellipticity, symmetry and positivity.

$\mathrm{H}(\mathcal{C}) \quad \mathcal{C}: Q \times \mathbb{S}^{d} \rightarrow \mathbb{S}^{d}$ is a viscosity tensor, $\mathcal{C}(t)=\left\{a_{i j k l}(t)\right\}$, such that $a_{i j k l}=a_{k l i j}=a_{i j l k} \in L^{\infty}(Q)$ and there exists $m_{1}>0$ satisfying $\mathcal{C}(t) \tau: \tau \geq m_{1}\|\tau\|_{\mathbb{S}^{d}}^{2}$ for all $\tau \in \mathbb{S}^{d}$, a.e. in $Q$.

$\mathrm{H}(\mathcal{G}) \quad \mathcal{G}: \Omega \times \mathbb{S}^{d} \rightarrow \mathbb{S}^{d}$ is an elasticity tensor, $\mathcal{G}=\left\{b_{i j k l}\right\}$, such that $b_{i j k l}=b_{k l i j}=b_{i j l k} \in L^{\infty}(\Omega)$ and $\mathcal{G} \tau: \tau \geq 0$ for all $\tau \in \mathbb{S}^{d}$, a.e. in $\Omega$.

Next, we define the operators $A(t), B \in \mathcal{L}\left(V, V^{*}\right)$ by

$$
\langle A(t) u, v\rangle=\langle\mathcal{C}(t) \varepsilon(u), \varepsilon(v)\rangle_{L^{2}\left(\Omega ; \mathbb{S}^{d}\right)}, \quad\langle B u, v\rangle=\langle\mathcal{G} \varepsilon(u), \varepsilon(v)\rangle_{L^{2}\left(\Omega ; \mathbb{S}^{d}\right)}
$$

for $t \in(0, T)$ and $u, v \in V$, and $f \in \mathcal{V}^{*}$ by (16). Analogously as in the first contact problem, we derive the following hemivariational inequality which is a weak form of (20a)-(20g): find $u:(0, T) \rightarrow V$ such that $u \in L^{\infty}(0, T ; V), u^{\prime} \in V$ and

$$
\left\{\begin{array}{l}
\left\langle A(t) u^{\prime}(t)+B u(t), v\right\rangle+\int_{\Gamma_{C}}\left(j_{v}^{0}\left(t, u_{v} ; v_{v}\right)+j_{\tau}^{0}\left(t, u_{\tau} ; v_{\tau}\right)\right) d \Gamma \geq\langle f(t), v\rangle \quad \text { for a.e. } t \in(0, T) \quad \text { and all } v \in V \\
u(0)=u_{0} .
\end{array}\right.
$$

Let us consider the functional $J:(0, T) \times X \rightarrow \mathbb{R}$ defined by

$$
J(t, v)=\int_{\Gamma_{C}}\left(j_{v}\left(x, t, v_{v}(x)\right)+j_{\tau}\left(x, t, v_{\tau}(x)\right)\right) d \Gamma \quad \text { for a.e. } \quad t \in(0, T) \quad \text { and all } \quad v \in X,
$$

where $X=L^{2}\left(\Gamma_{C} ; \mathbb{R}^{d}\right)$. 


\section{Lemma 6.4.}

Under the hypotheses VIII and IX, the functional J defined by (23) satisfies

(i) $J(\cdot, v)$ is measurable for all $v \in X$ and $J(\cdot, 0) \in L^{1}(0, T)$;

(ii) $J(t, \cdot)$ is Lipschitz on bounded subsets of $X$;

(iii) $\|\partial J(t, v)\|_{X^{*}} \leq \bar{c}\left(1+\|v\|_{X}\right)$ for all $v \in X$, a.e. $t \in(0, T)$ with $\bar{c}=2 \sqrt{2} \max \left\{c_{v}, c_{\tau}\right\} \max \left\{1, \sqrt{m\left(\Gamma_{C}\right)}\right\}$;

(iv) for all $v, w \in X$, we have

$$
J^{0}(t, v ; w) \leq \int_{\Gamma_{C}}\left(j_{v}^{0}\left(t, v_{v} ; w_{v}\right)+j_{\tau}^{0}\left(t, v_{\tau} ; w_{\tau}\right)\right) d \Gamma,
$$

where $J^{0}(t, v ; w)$ denotes the directional derivative of $J(t, \cdot)$ at a point $v \in X$ in the direction $w \in X$. If, in addition,

$$
\text { either } j_{v}(x, t, \cdot), j_{\tau}(x, t, \cdot) \text { are regular or }-j_{v}(x, t, \cdot),-j_{\tau}(x, t, \cdot) \text { are regular, }
$$

then either $J(t, \cdot)$ or $-J(t, \cdot)$ is regular, respectively and (24) holds with equality.

Under our notation we associate with the hemivariational inequality (22) the following inclusion of type (7): find $u \in L^{\infty}(0, T ; V)$ with $u^{\prime} \in \mathcal{V}$ such that

$$
\left\{\begin{array}{l}
A(t) u^{\prime}(t)+B u(t)+\gamma^{*} \partial J(t, \gamma u(t)) \ni f(t) \quad \text { for a.e. } \quad t \in(0, T) \\
u(0)=u_{0}
\end{array}\right.
$$

\section{Remark 6.5.}

We notice that if the hypotheses VIII and IX hold, then every solution to (26) is a solution to (22). The converse holds provided $j_{v}$ and $j_{\tau}$ satisfy the regularity condition (25). These facts follow from the definition of the Clarke subdifferential and Lemma 6.4 (iv).

The existence and regularity result for the hemivariational inequality (22) reads as follows.

\section{Theorem 6.6.}

If the hypotheses $\mathrm{H}(\mathrm{C}), \mathrm{H}(\mathcal{G}),(\mathrm{VIII.a})-(\mathrm{VIII.c}),\left(\right.$ IX.a)-(IX.c), (11), (12) hold and $m_{1}>2 \bar{c} T c_{e}^{2}\|\gamma\| \max \{1,\|\gamma\|\}$, where $\bar{c}$ is

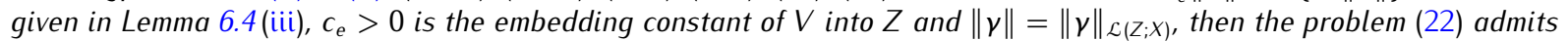
at least one solution $u \in L^{\infty}(0, T ; V)$ with $u^{\prime} \in \mathcal{V}$.

Proof. It follows from $\mathrm{H}(\mathcal{C})$ and $\mathrm{H}(\mathcal{G})$ that the operators $A(t)$ and $B$ defined by (21) satisfy $\mathrm{H}(A)$ with $\alpha=m_{1}$ and $\mathrm{H}(B)$, respectively. It is a consequence of Lemma 6.4 (i)-(iii) that the functional J given by (23) satisfies $\mathrm{H}(J)$. Also $\mathrm{H}(M)$ follows easily by the properties of the trace operator. The conclusion follows from Theorem 4.2 and Remark 6.5.

We conclude this section with short comments on multivalued boundary conditions (20e) and (20f) which are met in solid mechanics. The condition (20e) is a generalization of a normal compliance condition to the nonmonotone setting. Let the function $j_{v}: \mathbb{R} \rightarrow \mathbb{R}$ be defined by $j_{v}(r)=\int_{0}^{r} p_{v}(s) d s$, where for simplicity we drop the $(x, t)$-dependence and the function $p_{v} \in L_{\text {loc }}^{\infty}(\mathbb{R})$ is such that $\left|p_{v}(s)\right| \leq p_{1}(1+|s|)$ for $s \in \mathbb{R}$ with $p_{1}>0$ and $\operatorname{limits}_{\lim _{r \rightarrow s \pm}} p_{v}(r)$ exist for all $s \in \mathbb{R}$. It is well known, see e.g. $[16,18]$, that $\partial j_{v}(r)=\widehat{p}_{v}(r)$ for $r \in \mathbb{R}$, where the multivalued function $\widehat{p}_{v}: \mathbb{R} \rightarrow 2^{\mathbb{R}}$ is given by $\widehat{p}_{v}(r)=\left[p_{v}^{(1)}(r), p_{v}^{(2)}(r)\right],[\cdot, \cdot]$ denotes an interval in $\mathbb{R}$ and

$$
p_{v}^{(1)}(r)=\lim _{\varepsilon \rightarrow 0^{+}} \operatorname{essinf} p_{|\tau-r| \leq \varepsilon}(\tau), \quad p_{v}^{(2)}(r)=\lim _{\varepsilon \rightarrow 0^{+}} \underset{|\tau-r| \leq \varepsilon}{\operatorname{ess} \sup } p_{v}(\tau)
$$

In this case VIII holds and (20e) has the form $-\sigma_{v} \in \widehat{p}_{v}\left(u_{v}\right)$ on $\Gamma_{C} \times(0, T)$. Modifications of this example describe the nonmonotone zigzag relations in contact laws for a granular material and a reinforced concrete, cf. [18, Sections 2.4 and 7.2], 
[16, Section 4.6] and [12]. We also observe that if, in addition, $p_{v}$ is a continuous function, then (20e) reduces to $-\sigma_{v}=p_{v}\left(u_{v}\right)$ on $\Gamma_{C} \times(0, T)$ which is a well-known classical normal compliance condition. The normal compliance contact condition describes a reactive foundation. It assigns a reactive normal pressure which depends on the interpenetration of the asperities on the boundary surface and those of the foundation. We mention that the normal compliance condition is considered in engineering and certain mathematical publications as an approximation of the Signorini nonpenetration condition, and this idealization makes sense for practical reasons, cf. [19].

In the friction condition (20f), if $j_{\tau}=0$, we obtain the frictionless contact. If $j_{\tau}(x, t, \xi)=S(x, t)\|\xi\|_{\mathbb{R}^{d}}$, where $S \in L^{\infty}\left(\Gamma_{C} \times(0, T)\right)$ and $S>0$ a.e., then we get a version of the static Tresca friction law where the friction bound depends on time. In the friction condition (20f) we suppose that the tangential shear on the contact surface is given as a nonmonotone, time dependent and possibly multivalued function of the tangential displacement. For other examples of functions $j_{v}$ and $j_{\tau}$, we refer to [12]. Other two- and three-dimensional nonconvex zigzag superpotential laws are detailed in $[15,16]$.

\section{Acknowledgements}

This work has been supported by the National Science Centre of Poland under Grant no. N N 201604640 and by the Marie Curie International Research Staff Exchange Scheme Fellowship within the 7th European Community Framework Programme under Grant Agreement no. PIRSES-GA-2011-295118.

\section{References}

[1] Clarke F.H., Optimization and Nonsmooth Analysis, Canad. Math. Soc. Ser. Monogr. Adv. Texts, John Wiley \& Sons, New York, 1983

[2] Denkowski Z., Migórski S., Papageorgiou N.S., An Introduction to Nonlinear Analysis: Theory, Kluwer, Boston, 2003

[3] Denkowski Z., Migórski S., Papageorgiou N.S., An Introduction to Nonlinear Analysis: Applications, Kluwer, Boston, 2003

[4] Duvaut G., Lions J.-L., Inequalities in Mechanics and Physics, Grundlehren Math. Wiss., 219, Springer, Berlin-New York, 1976

[5] Eck C., Jarušek J., Krbec M., Unilateral Contact Problems, Pure Appl. Math. (Boca Raton), 270, Chapman Hall/CRC, Boca Raton, 2005

[6] Han W., Sofonea M., Quasistatic Contact Problems in Viscoelasticity and Viscoplasticity, AMS/IP Stud. Adv. Math., 30, American Mathematical Society, Providence, 2002

[7] Jarušek J., Dynamic contact problems with given friction for viscoelastic bodies, Czechoslovak Math. J., 1996, 46(121)(3), 475-487

[8] Jarušek J., Eck C., Dynamic contact problems with small Coulomb friction for viscoelastic bodies. Existence of solutions, Math. Models Methods Appl. Sci., 1999, 9(1), 11-34

[9] Migórski S., Dynamic hemivariational inequality modeling viscoelastic contact problem with normal damped response and friction, Appl. Anal., 2005, 84(7), 669-699

[10] Migórski S., Evolution hemivariational inequality for a class of dynamic viscoelastic nonmonotone frictional contact problems, Comput. Math. Appl., 2006, 52(5), 677-698

[11] Migórski S., Ochal A., Hemivariational inequality for viscoelastic contact problem with slip-dependent friction, Nonlinear Anal., 2005, 61(1-2), 135-161

[12] Migórski S., Ochal A., A unified approach to dynamic contact problems in viscoelasticity, J. Elasticity, 2006, 83(3), 247-275

[13] Migórski S., Ochal A., Quasi-static hemivariational inequality via vanishing acceleration approach, SIAM J. Math. Anal., 2009, 41(4), 1415-1435 
[14] Migórski S., Ochal A., Sofonea M., History-dependent subdifferential inclusions and hemivariational inequalities in contact mechanics, Nonlinear Anal. Real World Appl., 2011, 12(6), 3384-3396

[15] Migórski S., Ochal A., Sofonea M., Nonlinear Inclusions and Hemivariational Inequalities, Adv. Mech. Math., 26, Springer, New York, 2012

[16] Naniewicz Z., Panagiotopoulos P.D., Mathematical Theory of Hemivariational Inequalities and Applications, Monogr. Textbooks Pure Appl. Math., 188, Marcel Dekker, New York, 1995

[17] Panagiotopoulos P.D., Inequality Problems in Mechanics and Applications, Birkhäuser, Boston, 1985

[18] Panagiotopoulos P.D., Hemivariational Inequalities, Springer, Berlin, 1993

[19] Shillor M., Sofonea M., Telega J.J., Models and Analysis of Quasistatic Contact, Lecture Notes in Phys., 655, Springer, Berlin, 2004

[20] Sofonea M., Rodríguez-Arós A., Viaño J.M., A class of integro-differential variational inequalities with applications to viscoelastic contact, Math. Comput. Modelling, 2005, 41(11-12), 1355-1369

[21] Zeidler E., Nonlinear Functional Analysis and its Applications, II/B, Springer, New York, 1990 